\title{
From the lab to real-world use
}

\author{
A significant amount of research is needed to determine whether promising technologies in the lab could translate \\ into implementable solutions to achieve sustainability.
}

E xciting new sustainability-relevant technologies are being developed in chemistry, materials science, engineering and other research domains around the world. These promising technologies cover diverse areas that include, for example, converting sewage sludge into biofuels or using magnetic nanoparticles to remove pesticides from drinking water. The majority of these studies are performed on a small scale in the laboratory under strict conditions of cleanliness, temperature and pressure, and using solutions of predetermined chemical concentrations. These controlled conditions are needed for developing new methods, studying the effects of multiple variables, and determining the mechanisms underlying chemical or biological processes. However, these initial studies are quite far from the implementation of the related technologies that could eventually be used to achieve a more sustainable society.

One of the first steps towards exploring the real-world potential of lab-scale technologies is to test how well they operate under real-world conditions. This includes moving lab experiments outdoors, where effects from daylight and weather changes can be studied. It also includes using realworld samples. For example, before a system utilizing desalination to produce drinking water from seawater is tested on seawater, it generally undergoes initial tests using lab-mixed solutions containing known concentrations of sodium chloride and other salts. These known solutions are simple to obtain and can be reliably reproduced to ensure that additional experiments are performed under the same conditions. Later, the desalination system's robustness may be tested using seawater from several locations with differing salinities and trace metal contents. However, these movements towards the use of real-world conditions are just the first steps towards scale-up.

When researching sustainable technologies, lab-scale studies are often performed with small volumes, weights or sizes of materials. For example, technologies using liquids are likely to use volumes ranging from a few hundred millilitres up to a few hundred litres. Meanwhile, their related industrial-scale processes are more likely to be thousands of gallons or more. However, most lab-scale technologies

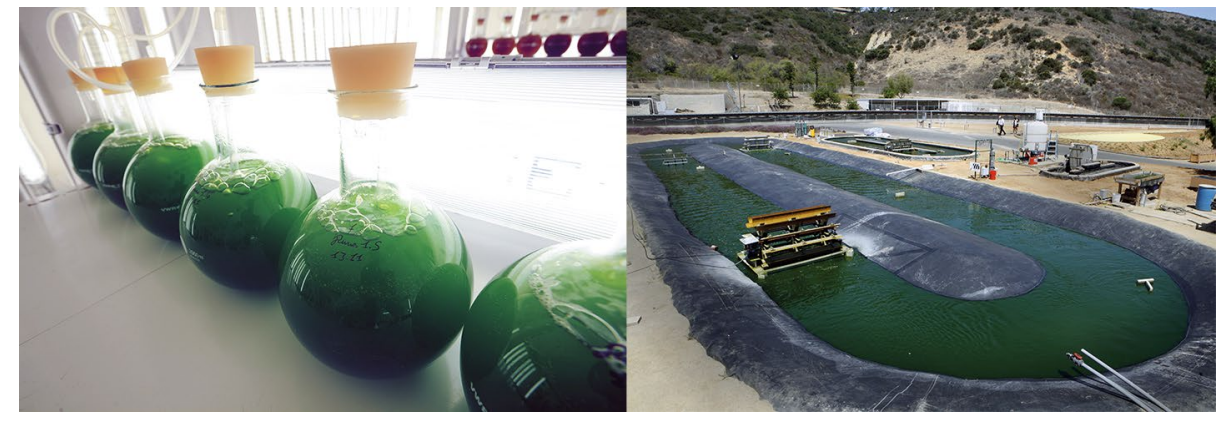

Credit: XAVIER LEOTY/AFP/Getty Images (left) and Mark Boster/Los Angeles Times via Getty Images (right)

cannot be translated directly into full-scale technologies simply by using larger volumes of chemicals and larger equipment sizes. For example, microalgae are studied for potential production of biofuels or healthrelated products like omega-3 fatty acid supplements. Microalgae cells settle at the bottom of their container, where only the surface cells receive adequate light exposure. To overcome this, lab experiments may be performed in a flask mixed by gentle stirring or bubbling to keep the cells suspended for improved light exposure to increase growth rates. However, on an industrial scale, microalgae are often grown in open raceway ponds mixed by paddlewheels. Fluid movement differs in a small flask compared to a large pond, which affects the efficiency of mixing and may result in dead zones where cells settle and accumulate. Cells in dead zones will multiply more slowly due to reduced light exposure, and open ponds are exposed to contamination by microorganisms that may outcompete or consume the microalgae. These changes will reduce microalgae growth rates and alter oil contents compared to growth under controlled laboratory conditions, so labscale studies that show promising biofuel or nutrient production may be unsuccessful at larger scales. These are just a few reasons why successfully scaling-up a technology from experiments in a laboratory to a pilot or a full-scale process involves a great deal of additional research to overcome problems that hinder the implementation of promising technologies.

Part of Nature Sustainability's mission is to highlight promising solutions to the most pressing environmental and societal challenges, and in order to accomplish this, we encourage relevant submissions of applied research and engineering studies. In an effort to show how to move promising sustainability-related technologies out of the lab and into real-world use, we are especially interested in larger-scale, longerterm studies using real-world conditions. In a recently published Analysis, Afzal et al. studied the effectiveness of floating treatment wetlands, which use a mixture of plants on floating rafts to treat wastewater. Over three years, the authors studied the effectiveness of full-scale floating treatment wetlands at improving water treatment in stabilization ponds used by the city of Faisalabad, Pakistan, to treat a mixture of sewage and industrial wastewater. The long timeframe allowed the authors to compare the effects of plant biomass on nutrient removal and to consider differing wastewater levels over time. This research demonstrates the effectiveness of a simple, low-cost option for improving wastewater treatment in existing wastewater retention ponds. Studies like the one by Afzal and colleagues can help bridge the gap between the laboratory and real-world implementation by presenting valuable research on potential sustainability solutions under real-world conditions. We look forward to receiving more of such studies covering various environmental challenges and hope that our readers will agree with us about the scholarly merit and societal value of this kind of applied research.

Published online: 12 November 2019 https://doi.org/10.1038/s41893-019-0435-7 